\title{
Optimization of a Phosphorus-doped Silica Sol Coating on Cotton Fabrics
}

\author{
Jia-Li GU ${ }^{\mathrm{a}}$, Qiang-Hua ZHANG ${ }^{\mathrm{b}}$, Yun-Bo $\mathrm{CHEN}^{\mathrm{c}}$, Guo-Qiang $\mathrm{CHEN}^{\mathrm{d}}$ and \\ Tie-Ling XING ${ }^{\mathrm{e},}$ \\ ${ }^{1}$ National Engineering Laboratory for Modern Silk, Soochow University, Suzhou, China \\ 2 Jiangsu HuaJia Group, Suzhou, China \\ *xingtieling@suda.edu.cn
}

Keywords: Phosphorus-doped, Cotton, Coating, Flame retardancy, Water repellence.

\begin{abstract}
The pure silica hydrosol and phosphorus-doped silica hydrosol was prepared using sol-gel method with methyltrimethoxylsilane(MTMOS) and tetraethoxysilane(TEOS) as precursors and ammonium biphosphate as a flame retardant(FR) in the presence of hydrochloric acid and cationic /nonionic surfactants in aqueous solution. Then the synthesized sol was modified by hexadecyltrimethoxysilane(HDTMS) and applied to cotton fabric to endow the fabric with flame retardancy and low surface energy. The effects of different conditions on flame retardancy and hydrophobicity of cotton fabric were discussed. The factors about dosage of precursors and FR, the ratio of surfactants, $\mathrm{pH}$ were determined. The Limited Oxygen Index (LOI), contact angle (CA) test and thermogravimetric analysis were used to indicate differences between treated and untreated cotton fabrics. The results show that the phosphorus-doped silica coating is helpful to improve the flame retardancy and water repellence of cotton fabrics.
\end{abstract}

\section{Introduction}

In human's daily life, cotton as a high production natural fiber plays a significant role on costume attributed to its outstanding wear abilities such as comfortableness, hygroscopicity and air permeability. However, the inflammability of cotton restricted its application in functional textiles area. Additionally, excellent hydrophobicity can meet a self-cleaning demand $[1,2]$. Accordingly, it is a feasible direction to improve additional value of cotton fabric. Generally, there are two approaches to achieve hydrophobicity of fabric: decreasing surface energy and increasing roughness of fabric surface. The former relies on the modification of fabric surface by introducing long chain substance or fluorine-contained compounds [3, 4]. The latter can be obtained by attaching nanoparticles on fabric surface through sol-gel method [5]. In terms of flame retardancy, phosphorus, boron and nitrogen as flame resistant elements are regularly applied in fabric treatment [6-8]. In recent years, sol-gel method has been largely employed in surface modification of fabric to improve flame retardancy.

It is reported that sol-gel method is an micro/nano scale technology with simple process and low reaction temperature, which has been largely used in preparing organic, inorganic or hybrid materials [9, 10]. Jenny Alongi team [11, 12] did large work about enhancing flame retardancy of different kinds of fabrics with sol-gel method. However, most of these investigations were using ethanol as solvent which is expensive, flammable and may cause pollution problem. In this study, ethanol was replaced by distilled water as solvent during the sol preparation. Surfactant was used to disperse organic phase into water system. And the optimal sol synthesis and fabric treatment conditions were determined. 


\section{Experimental}

\section{Materials and Reagents}

Twill-Weave cotton fabric $\left(308 \mathrm{~g} / \mathrm{m}^{2}\right)$ was provided from Jiangsu Shayin Group. Tetraethoxysilane (TEOS), polysorbate 60 (Tween 60), cetyltrimethylammonium chloride (CTAC) were purchased from Sinopharm Chemical Reagent Co. Ltd. Methyltrimethoxylsilane(MTMOS), hexadecyltrimethoxysilane (HDTMS), ammonium biphosphate (FR) were purchased from Aladdin Inc.

\section{Hydrosol Preparation and Cotton Treatment}

Firstly, MTMOS was dispersed in 100mL distilled water by Lab Hishaer Disperser (FA25, FLUKO Inc, German) in the presence of surfactants. Then FR was dissolved in above mixture. Then MTMOS was hydrolyzed in acid condition for $4 \mathrm{~h}$, afterwards TEOS was added drop by drop and reacted for $2 \mathrm{~h}$. Hereto the hydrosol was completely prepared. Finally, HDTMS was used as a modification agent to obtain water repellency of cotton.

Cotton fabric was treated by pad-dry-cure method using Vertical Padder (FL-400, Shanghai Longcolor Inc.) at a nip pressure of $2 \mathrm{Kg} / \mathrm{cm}^{2}$ and dried at $80^{\circ} \mathrm{C}$ for $5 \mathrm{~min}$, then cured at certain temperature for $5 \mathrm{~min}$ by Heat-setting Machine (LD-360B, Shanghai Longcolor Inc). Subsequently, the treated samples were placed at a standard atmosphere $\left\{25 \pm 2^{\circ} \mathrm{C} ; 65 \%\right.$ humidity $\}$ condition for 24 hours before tests.

\section{Test Methods}

\section{Limited Oxygen Index (LOI) Measurement}

LOI measurement was followed by GB/T5454-1997 (Fire Testing Technology Inc, England).

\section{Contact angle Measurement}

Cotton fabric was put on the OCA contact angle instrument (Dataphysics Inc) for test. The value was recorded after 3s when water drop on cotton surface. Each sample was tested at three points and on the average of three measurements.

\section{Thermogravimetric Analysis}

TG was measured by Thermal Analyzer (DIAMOND 5700, PE Ltd USA). The sample approximately $5 \mathrm{mg}$ in weight, was introduced into an open alumina sample pan and was heated from $50{ }^{\circ} \mathrm{C}$ to $600{ }^{\circ} \mathrm{C}$ at a heating rate of $10^{\circ} \mathrm{C} / \mathrm{min}$ in nitrogen atmosphere.

\section{Results and Discussions}

\section{Effect of Proportion of Surfactant on LOI and CA Value}

In this experiment, cetyltrimethylammonium chloride (CTAC) was used as a cationic surfactant and polysorbate 60(Tween60) as a nonionic surfactant. It can be seen from Fig. 1 that with the proportion of nonionic surfactant increasing, the LOI value was decreased slightly. In addition, the CA values were relatively steady. During the test, it can be found that the remained time of water drop on cotton surface was increased with the increase of Tween60. Thus, the presence of Tween60 had a beneficial effect on improving hydrophobicity of cotton fabric. Taking sol stability into consideration, 2:3 of CATA: Tween60 was chosen as the optimal ratio of surfactants. At this proportion, CA value reached the highest value of $139.0^{\circ}$. 


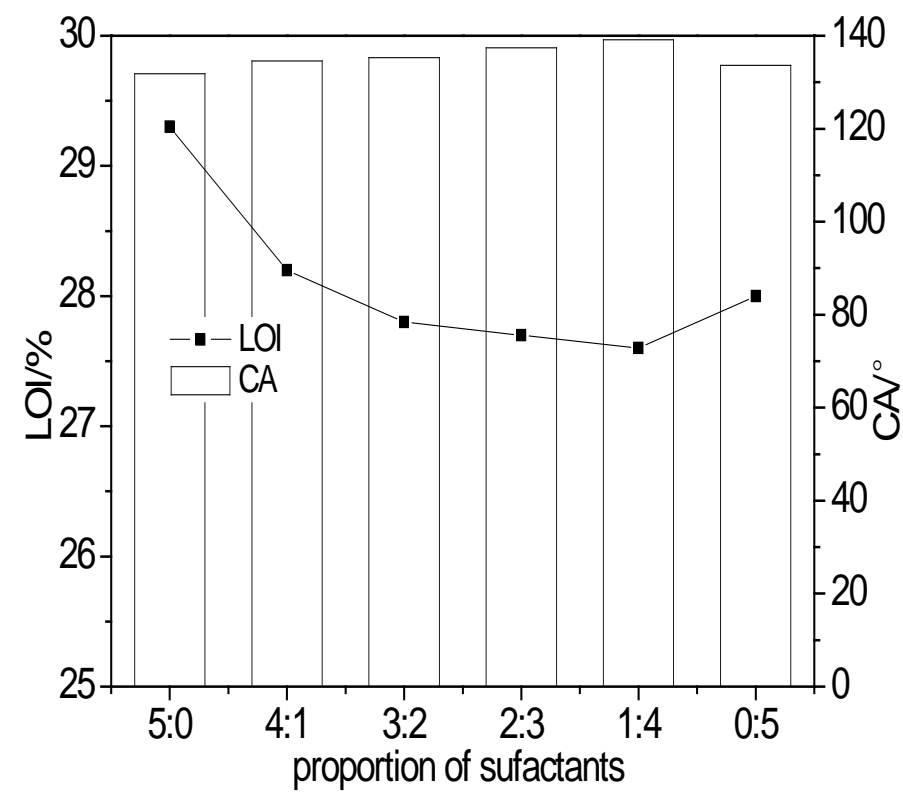

Fig. 1 Influence of proportion of surfactant on LOI and CA of cotton fabric

Note: proportion is the ratio of CTAC and Tween 60 ; synthesis temperature $30^{\circ} \mathrm{C}$; $\mathrm{pH} 3$; dosage of surfactant, TEOS, MTMOS, FR were $0.9 \%$, $2.5 \mathrm{~g}, 1.5 \mathrm{~g}, 8 \mathrm{~g}$; dried at $80^{\circ} \mathrm{C}$ for $5 \mathrm{~min}$, cured at $130^{\circ} \mathrm{C}$ for $5 \mathrm{~min}$.

\section{Effect of Precursors Dosage on LOI and CA Value}

In Fig. 2, it can be seen that the LOI value increased with the amount of TEOS increasing. As was known in literature [13], TEOS was largely used with other flame retardant elements such as phosphorus, boron and nitrogen in sol-gel method to obtain the flame resistant textiles. Ethoxyl in TEOS would be hydrolyzed into hydroxyl in the presence of acid, and the hydroxyl suffered a dehydration condensation process to form Si-O-Si structure, which could also enhance the roughness on fabric surface so that the hydrophobicity of treated fabrics would be improved at the same. The result could be proved in Fig. 2 that the CA value was improved from 107.4 to $135.5^{\circ}$ while TEOS dosage from 0 to $2.5 \mathrm{~g}$.

As shown in Fig. 3, with the increase of MTMOS dosage, the LOI value decreased slightly in general. This phenomenon maybe due to the existence of flammable group $-\mathrm{CH}_{3}$ in MTMOS. When the MTMOS dosage was increased, although the content of silicon was increased, the content of $-\mathrm{CH}_{3}$ group were also increased. In the experiment step, the precipitation appeared when the additive amount was up to $3 \mathrm{~g}$ indicating that the synthesis system reached the limit. MTMOS was used to enhance hydrophobicity because of the existence of hydrophobic groups $-\mathrm{CH}_{3}$. Therefore, with the increase of MTMOS, CA value was improved. Accordingly, the dosage of MTMOS was chosen as 1.5g. 


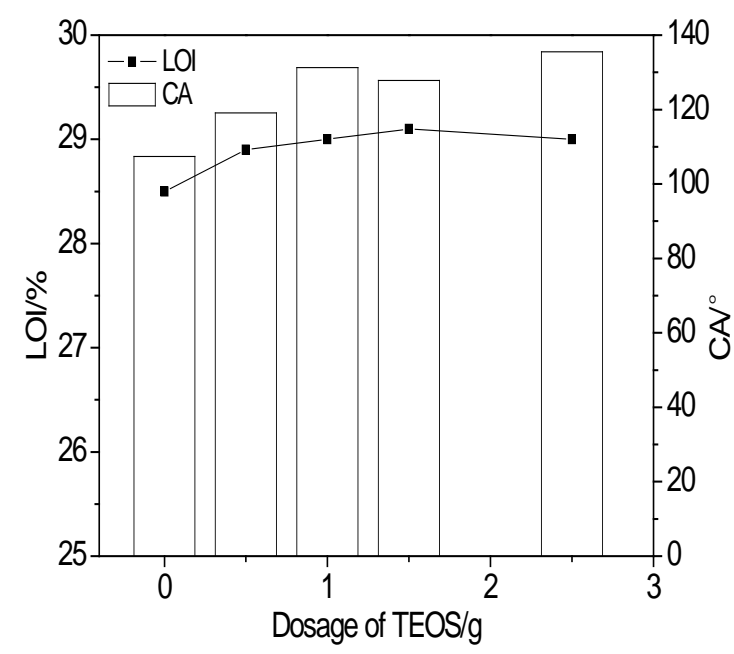

Fig. 2 Influence of dosage of TEOS

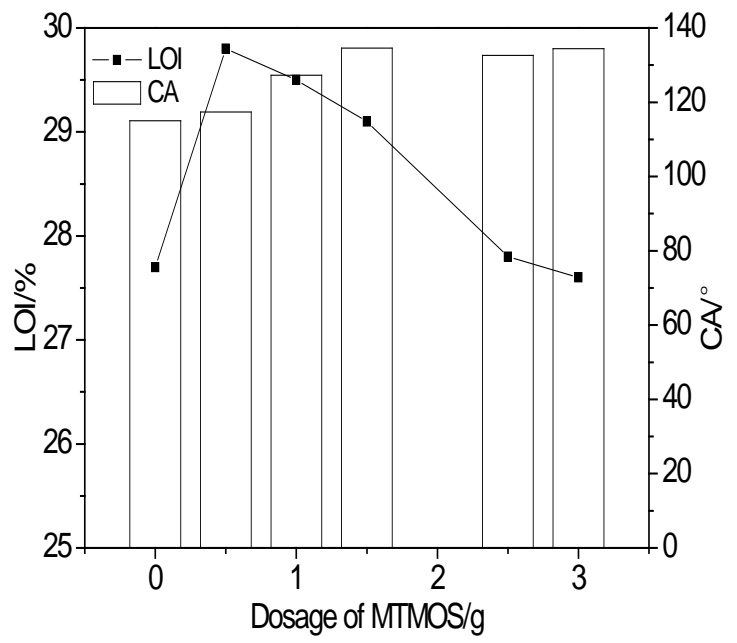

Fig. 3 Influence of dosage of MTMOS

Note: synthesis temperature $30^{\circ} \mathrm{C}$; $\mathrm{pH} 3$; dosage of surfactant, FR were $0.9 \%$, 8g; CTAC:Tween $=2: 3$; dried at $80^{\circ} \mathrm{C}$ for $5 \mathrm{~min}$, cured at $130^{\circ} \mathrm{C}$ for $5 \mathrm{~min}$.

\section{Effect of Synthesis pH on LOI and CA Value}

The acid environment was used as the sol preparation condition. It can be seen from Fig. 4 that the LOI value decreased while the $\mathrm{pH}$ value increased. It is harmful for cotton fabric at too low $\mathrm{pH}$ value because cotton fabric is not resistant to acid and the strength of cotton fabric might be damaged heavily under strong acidic condition. Besides, at lower $\mathrm{pH}$ value, the cotton fabric would appear yellowing and a hard handle would be obtained. Although a better LOI value was obtained at lower $\mathrm{pH}, \mathrm{pH} 3$ was still chosen as the feasible $\mathrm{pH}$ value.

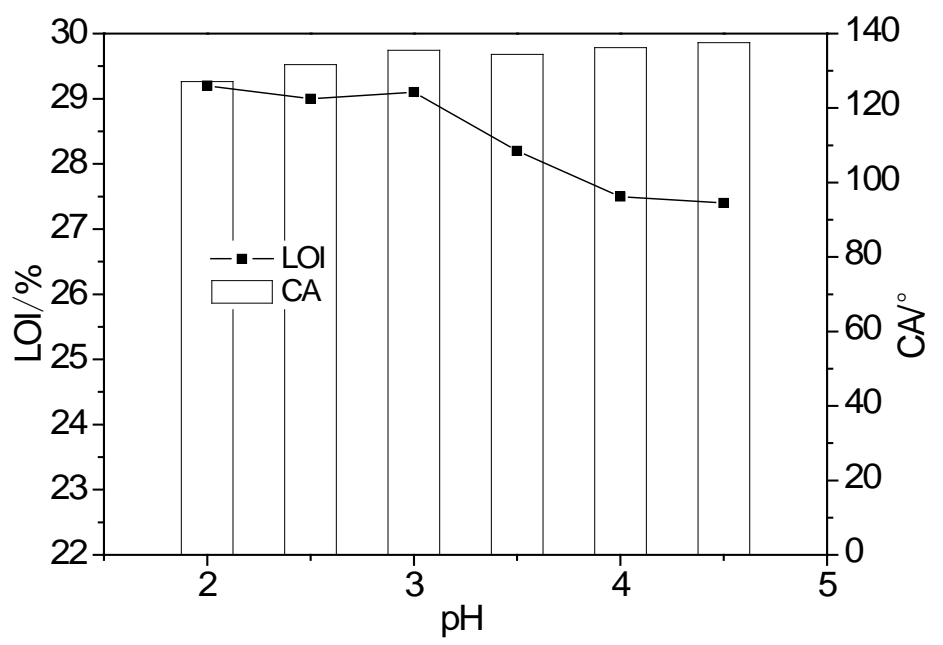

Fig. 4 Influence of synthesis $\mathrm{pH}$

Note: synthesis temperature $30^{\circ} \mathrm{C}$; dosage of surfactant, TEOS, MTMOS, FR were $0.9 \%, 2.5 \mathrm{~g}, 1.5 \mathrm{~g}$, 8g; CTAC:Tween $=2: 3$; dried at $80^{\circ} \mathrm{C}$ for $5 \mathrm{~min}$, cured at $130^{\circ} \mathrm{C}$ for $5 \mathrm{~min}$.

\section{Effect of Dosage of FR on LOI and CA Value}

As shown in Fig. 5, with the increase of FR dosage, LOI value showed an obvious rising trend. The flame resistance was improved greatly owing to the existence of phosphorus. There would be more phosphorus doped into the Si-O-Si structure while more FR addition in the synthesis process. During curing process, phosphorus element was attached onto cotton surface by physical adsorption. When cellulose suffered heating, the existence of phosphorus would release phosphate then 
promote the formation of char which formed a physical barrier to prevent cotton fabric from contacting with outside atmosphere and slowed down the phyrolysis reaction. As FR dosage increased from $8 \mathrm{~g}$ to $10 \mathrm{~g}$, the rising amplitude of LOI value had a slight decrease. In case of the dosage saving and sol stability, FR dosage was determined as $8 \mathrm{~g}$.

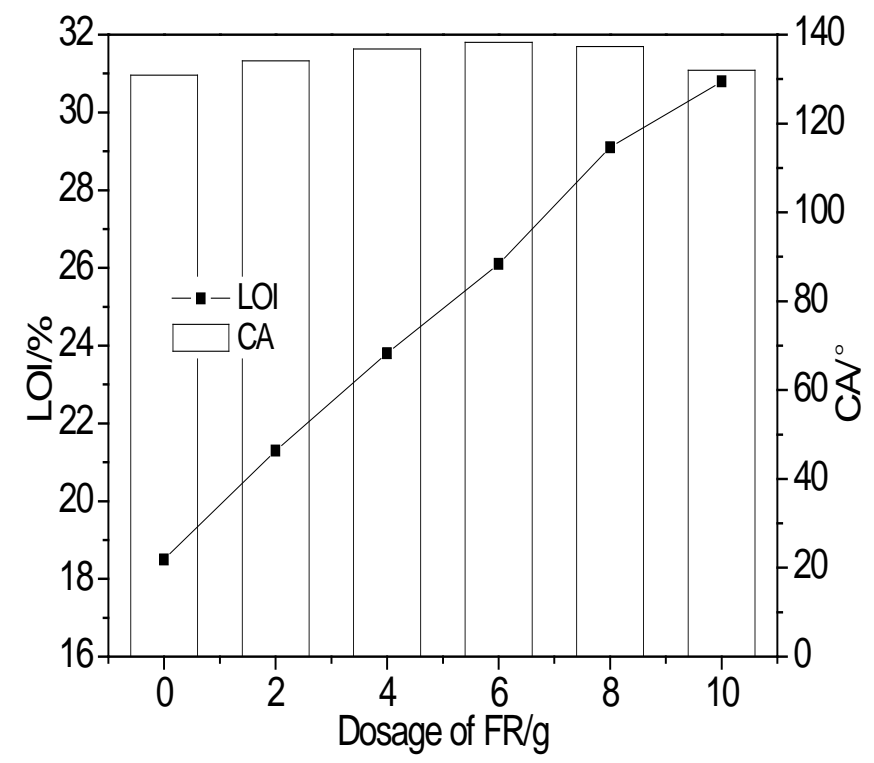

Fig. 5 Influence of dosage of FR on LOI and CA of cotton fabric

Note: synthesis temperature $30^{\circ} \mathrm{C}$; $\mathrm{pH} 3$; dosage of surfactant, TEOS, MTMOS were $0.9 \%, 2.5 \mathrm{~g}$, 1.5g; CTAC:Tween $=2: 3$; dried at $80^{\circ} \mathrm{C}$ for $5 \mathrm{~min}$, cured at $130^{\circ} \mathrm{C}$ for $5 \mathrm{~min}$.

\section{Thermal Property Analysis}

Fig. 7a and Fig. 7b were TG curves and DTG curves, respectively. Table 1 was TG data, LOI and contact angle data of cotton samples. Cot_control was pure cotton, cot_Si was treated with pure silica hydrosol, cot_Si/P was treated with phosphorus-doped silica hydrosol. From Fig. 7a and Table 1, it can be seen that compared with cot_control(13.3\%), cot_Si/P (29.7\%) has a vividly increase of weight residue . In Fig. 7b, the maximum weight loss rate of cot_control, cot_Si and cot_Si/P were $34.0 \%, 41.4 \%$ and $77.8 \%$ at $370^{\circ} \mathrm{C}, 338^{\circ} \mathrm{C}$ and $226^{\circ} \mathrm{C}$ of corresponding temperature, respectively. The above data were obviously illustrated that the phosphorus-doped hydrosol had an effective

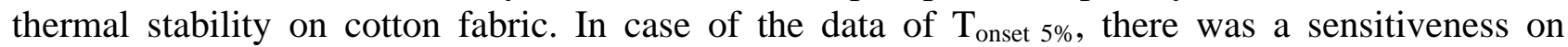
cot_Si $/ \mathrm{P}\left(65^{\circ} \mathrm{C}\right)$ and cot_Si $\left(47^{\circ} \mathrm{C}\right)$ compared with cot_control $\left(237^{\circ} \mathrm{C}\right)$ that is to say the starting temperature of weight loss was reduced compared with cot_control [14]. Additionally, cot_Si didn’t show a satisfactory result because the flammable long chains(HDTMS) was added into the hydrosol which would accelerate the degradation of cotton fabric. 


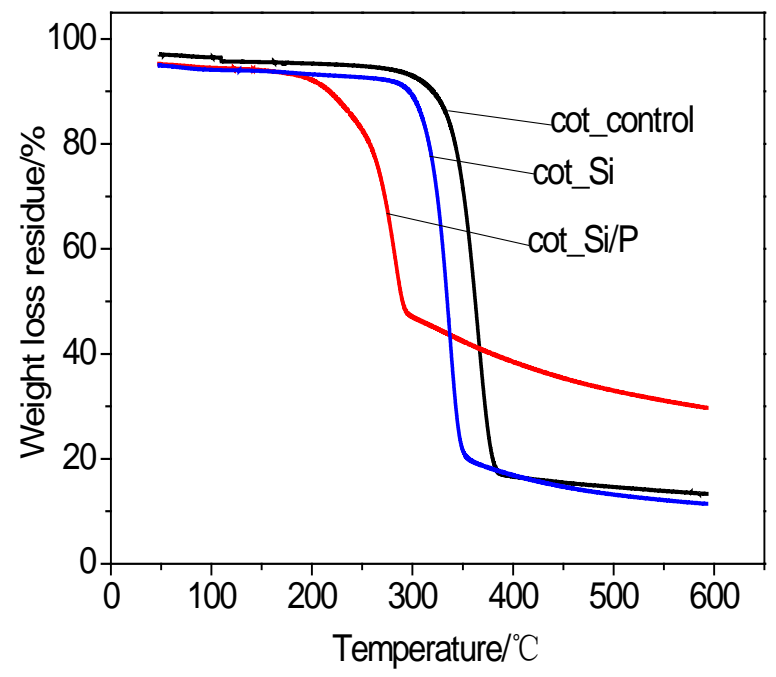

Fig. 7a TG curves of cotton samples

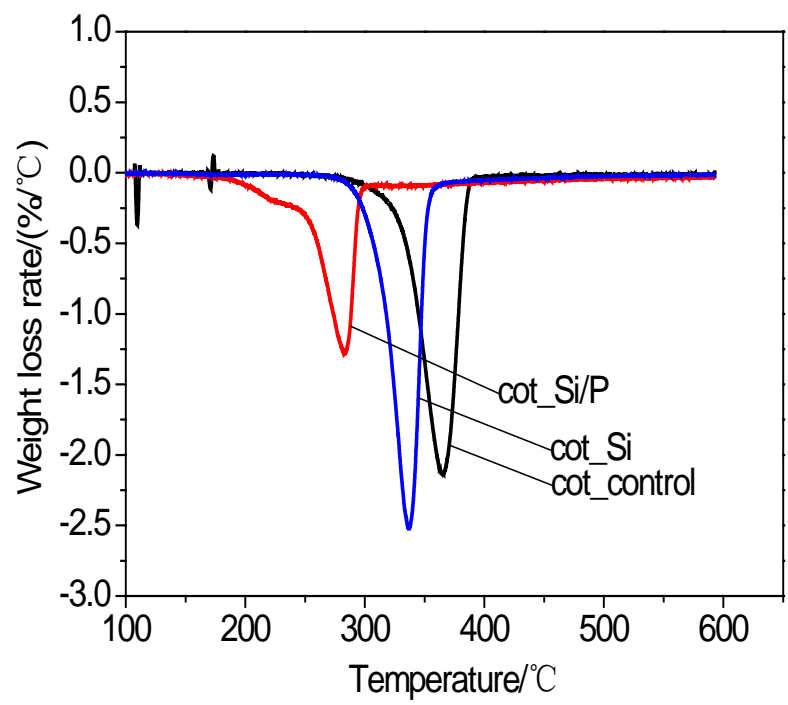

Fig. 7b DTG curves of cotton samples

Table 1 TG data and contact angle of cotton fabrics

\begin{tabular}{|c|c|c|c|c|c|c|}
\hline Sample & $\begin{array}{l}\mathrm{T}_{\text {onset }} \% \\
{\left[{ }^{\circ} \mathrm{C}\right]}\end{array}$ & $\begin{array}{l}\mathrm{T}_{\max } \\
{\left[{ }^{\circ} \mathrm{C}\right]}\end{array}$ & $\begin{array}{l}\text { Residue at } 600^{\circ} \mathrm{C} \\
{[\%]}\end{array}$ & $\begin{array}{l}\text { Residue at } \mathrm{T}_{\max } \\
\text { [\%] }\end{array}$ & $\begin{array}{l}\mathrm{LOI} \\
{[\%]}\end{array}$ & $\begin{array}{l}\text { Contact angle } \\
{\left[^{\circ}\right]}\end{array}$ \\
\hline cot_control & 237 & 370 & 13.3 & 34.0 & 18.5 & 1 \\
\hline cot_Si & 47 & 338 & 11.4 & 41.4 & 17.4 & 129.4 \\
\hline cot_Si/P & 65 & 262 & 29.7 & 77.8 & 29.2 & 135.2 \\
\hline
\end{tabular}

\section{Conclusion}

1. The phosphorus-doped hydrosol was synthesized successfully, and the optimized silica sol coating treatment were obtained: CTAC:Tween60=2:3, $\mathrm{pH} 3$, 1.5g MTMOS, $2.5 \mathrm{~g}$ TEOS, $8 \mathrm{~g}$ FR.

2. LOI value of treated cotton fabric was increased from $18.5 \%$ to $29.2 \%$. Thermogravimetric analysis results showed the residue of cotton fabric was largely increased from $34.0 \%$ to $77.8 \%$, indicating that phosphorus-doped silica hydrosol played a positive role on enhancing the flame retardancy and thermal stability of cotton fabric.

3. With the addition of HDTMS, cotton fabric surface displayed hydrophobicity, and CA value reached $135.2^{\circ}$. Therefore, HDTMS offered an improvement on hydrophobicity of treated cotton fabric. 


\section{Acknowledgement}

This work was supported by Natural Science Foundation of Jiangsu Province (BK20151242), Priority Academic Program Development of Jiangsu Higher Education Institutions (PAPD) and Qing Lan Project.

\section{References}

[1] M. Zhang, C.Y. Wang, Carbohydr. Polym. 96 (2013) 396-402.

[2] Y.J. Yin, C.X. Wang, Colloids Surf., A. 417 (2013) 120-125.

[3] L.H. Xu, W. Zhuang, B. Xu, Z.S. Cai, Appl. Surf. Sci. 257 (2011) 5491-5498.

[4] W.Q. Huang, Y.J. Xing, Y.Y. Yu, S.M. Shang, J.J. Dai, Appl. Surf. Sci. 257 (2011) 4443-4448

[5] M.P. Gashti, F. Alimohammadi, A. Shamei, Surf. Coat. Technol. 206 (2012) 3208-3215.

[6] Q.H. Zhang, W. Zhang, J.Y. Huang, Y.K. Lai, T.L. Xing, G.Q. Chen, W.R. Jin, H.Z. Liu, B. Sun, Mater. Des. 85 (2015) 796-799.

[7] J. Alongi, C. Colleoni, G. Rosace, G. Malucelli, J. Therm. Anal. Calorim. 110 (2012) 1207-1216.

[8] Z.Y. Yang, B. Fei, X.W. Wang, J.H. Xin, Fire. Mater. 36 (2012) 31-39.

[9] S.M. Shang, Z.X. Li, Y.J. Xing, J.H. Xin, X.M. Tao, Appl. Surf. Sci. 257 (2010) 1495-1499.

[10] Y.J. Yin, C.X. Wang, J. Sol Gel Sci Technol. 64 (2012) 743-749.

[11] J. Alongi, M. Ciobanu, G. Malucelli, Carbohydr. Polym. 87 (2012) 2093-2099.

[12] J. Alongi, G. Malucelli, J. Mater. Chem. 22 (2012) 21805-21809.

[13] K. Sevim, H. Zuhal, K.A. Nihan, G. Atilla, Prog. Org. Coat. 65 (2009) 49-55.

[14] J. Alongi, C. Colleoni, G Rosace, G. Malucelli, Polym. Degard. Stab. 99 (2014) 92-98. 